DOI: 10.1515/awutp -2015-0111

\title{
IMPROVING THE ACCURACY OF THE EMPIRICAL CLEAR SKY SOLAR IRRADIANCE MODELS
}

\author{
Oana Mares, Daniel Vizman, Marius Paulescu \\ Faculty of Physics, West University of Timisoara, Bd. V. Parvan 4, 300223 Timisoara, Romania
}

\section{Article Info}

Received: 29.03.2013

Accepted: 16.04.2013

Keywords: Solar irradiance, estimation, clear sky.

\begin{abstract}
This paper is focused on improving the quality of nowcasting solar irradiance by enhancing the clear sky component of the model. A simple linear correction for the average atmospheric transmittance is proposed. The correction is based on the most recent measurements recorded prior the forecasting moment. The proposed procedure was tested against data measured on the Solar Platform of the West University of Timisoara. Overall results demonstrates a notable improvement in the clear sky model accuracy.
\end{abstract}

\section{Introduction}

Some models for nowcasting solar irradiance include a component for estimating solar irradiance under clear sky conditions. These models are constructed empirically, usually by fitting long term data series recorded in situ. The fitted parameters are frozen and used, as they are, into the estimation model applied to future time moments. Since the solar irradiance forecasting models are applied on data measured in situ (generally the forecasting models contain autoregressive terms), the clear sky model can be finely adjusted prior to the forecasting moment.

In this paper, a procedure for improving the average atmospheric transmittance (as part of the simple empirical models for solar irradiance) is proposed. The adjustment is achieved by applying a dynamic correction. The correction is estimated with a simple linear regression on measured data in a period prior to the forecasting moment.

The paper is organized as follows. The next section is dedicated to the description of the relevant data used in this study. A measure for the state of the sky and the solar irradiance model are defined in Sec. 3. The computing procedure and its accuracy are discussed in Sec. 4. The last section contains the conclusions. 


\section{Database}

Global $\left(G_{t}\right)$ and diffuse $\left(G_{d, t}\right)$ solar irradiance recorded on the Solar Platform of the West University of Timisoara [1] are used in this study. The town of Timisoara (latitude $45^{0}$ $46^{\prime}$, $\mathrm{N}$, longitude $21^{0} 25^{\prime}$ ' $\mathrm{E}$ and $85 \mathrm{~m}$ asl) has a warm temperate climate, typical for the Pannonia Basin (Köppen climate classification $\mathrm{Cfb}$ ). This is based on the Kottek et al. digital Köppen-Geiger world map on climate classification, build with data from the second half of the 20 th century [2].

Measurements on the Solar Platform are performed all day long at equal time intervals of 15 seconds. DeltaOHM LP PYRA 02 first class pyranometers which fully comply with ISO 9060 standards and meet the requirements defined by the World Meteorological Organization are employed. The sensors are integrated into an acquisition data system based on National Instruments PXI Platform including a PXI-6259 data acquisition board.

Data recorded in a period of 5 days, from 15 to 20 July 2010, were used in this study. All measurements associated with the sun elevation angle $h<5^{\circ}$ have been removed from the database. There are two reasons for this: (i) the pyranometer's accuracy around sunrise and sunset is questionable and (ii) $\sin (h)$ tends towards zero for small values of $h$.

\section{Theory}

\subsection{Definition of the sunshine number}

For an observer placed on Earth's surface, the sunshine number $\xi(t)$ is defined as a time dependent random binary variable, as follows [3]:

$$
\xi_{t}=\left\{\begin{array}{l}
1 \quad \text { if the sun is shining at timet } \\
0 \text { otherwise }
\end{array}\right.
$$

Series of SSN values can be inferred from the series of measured solar irradiance values by using the World Meteorological Organization sunshine criterion. In our notation:

$$
\xi_{t}= \begin{cases}1 & \text { if }\left(G_{t}-G_{d, t}\right) / \sin h>120 \mathrm{~W} / \mathrm{m}^{2} \\ 0 & \text { otherwise }\end{cases}
$$

where $G_{t}$ and $G_{d, t}$ denote the global and diffuse solar irradiance at moment $t$, and $h$ is the sun's elevation angle. The average value of SSN over a given period $\Delta t$ equals the relative 
sunshine $\sigma$ during $\Delta t$. In order to quantify the stability of the solar radiative regime, a parameter related to SSN, the sunshine stability number (SSSN), was defined in Ref. [4]:

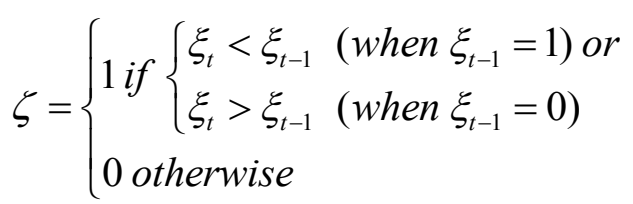

\subsection{Modeling global solar irradiance under clear sky conditions}

In this study, an empirical model fitted on data measured in Timisoara [5] was used. The model equation is:

$$
G_{0, t}=G_{e x t}\left[1-0.4645 \cdot e^{-0.69 \cdot \sin h_{z, t}}\right] \cdot e^{\frac{0.05211}{\sin h_{z, t}}} \cdot \sin h_{z, t}
$$

where $G_{\text {ext }}$ denotes the extraterrestrial solar irradiance (see e.g. [6] for details).

Equation (4) demonstrates a reasonable accuracy when was applied to estimate the amount of solar energy in Timisoara [5]. However, when Eq. (4) was tested for forecasting solar irradiance, a worsening of the prediction accuracy was noted. There are two main reasons for decreasing the model accuracy: (1) the clear sky states were selected by means of SSN $(\xi=1)$. SSN warrants that there are no clouds on the sun direction, but says nothing about the rest of the celestial vault. There may be clouds on the sky and the reflection on clouds increase the solar irradiance at the ground level. In this case Eq. (4) underestimates the measurements. (2) Due to the atmospheric circulation, the atmospheric transmittance is continuously changed. Sometimes the atmospheric transmittance can be substantially different from its average value encapsulated in Eq. (4). In such cases Eq. (4) may either underestimate or overestimate the measurements. The solution proposed in this paper consists in a simple dynamic correction:

$$
G=\tau_{a} \cdot G_{0, t}
$$

where $\tau_{a}$ is evaluated based on the most recent measurements with the sun shining on the sky $(\xi=1)$.

\section{Results and discussion}

The algorithm for estimating the cloud transmittance was implemented in MathCAD [7], software for scientific and engineering calculations. 
The model accuracy was assessed through the usual statistical indicators used in solar energy modeling: RMSE (root mean square error) and $M B E$ (mean bias error):

$$
R M S E=\left[\frac{1}{M} \sum_{i=1}^{M}\left(c_{t}-m_{t}\right)^{2}\right]^{1 / 2} \quad M B E=\frac{1}{M} \sum_{i=1}^{M}\left(c_{t}-m_{t}\right)
$$

where $c$ and $m$ refer to the computed and measured values, respectively, while $M$ is the sample size. In order to compare models quality applied to the sample with different magnitudes, these indicators are used normalized to the mean $\mu$ of the sample: $r R M S E$ $=R M S E / \mu$ and $r M B E=M B E / \mu$.

\subsection{Computational procedure}

Series of data sampled at 15 seconds are considered in this study. The estimation of solar irradiance was made using the empirical model given by Eq. (4). The model is applied only when the sun is shining $(\xi=1)$. The computational procedure is as follows: In the morning when $h=5^{\circ}$, the correction is initialized with the value $\tau_{a}=1$. At the next moment, if the sun is coved by clouds $(\xi=0)$, the algorithm do not perform any action and the correction preserve the previous value. If the sun is shining on the sky, $\tau_{a}$ is the estimated with a linear regression on the solar irradiance data measured in a period $\Delta t$. Only data in the state $\xi=1$ are considered. And so on, the algorithm is applied until evening; when $h$ reaches again $5^{\circ}$.

\subsection{Evaluation of the model accuracy}

Data recorded during $16^{\text {th }}$ to $20^{\text {th }}$ July 2010 were used to test the model. These days were selected because they have been characterized by different radiative regimes. Figure 1 shows the time series of global solar irradiation recorded in these days. Visual inspection shows that the sample includes both clear sky days and variable sky days. The first day, $16^{\text {th }}$ July, is a perfectly stable clear sky day $(\sigma=0.9236$ and $\bar{\zeta}=0.00088)$. Both last two days, $19^{\text {th }}$ and $20^{\text {th }}$ July, have unstable radiative regimes, $\left(\bar{\zeta}=0.00952\right.$ on $19^{\text {th }}$ July and $\bar{\zeta}=0.01134$ on $20^{\text {th }}$ July) but are completely different as far as the cloud cover is concerned. On $19^{\text {th }}$ July, the instability occurred on a mostly covered sky $(\sigma=0.1253)$, while on $20^{\text {th }}$ July the instability occurred on a mostly clear sky $(\sigma=0.6530)$.

The test procedure was conducted as follows. The correction $\tau_{a}$ was estimated at every 5 minutes based on using the twenty data measured in the previous interval ( $\Delta t=5 \mathrm{~min})$. The test was performed at three time intervals ahead (lead time): 15 seconds, 5 and 10 
minutes. Figure 2 presents a comparison between the estimated solar irradiance and the measured one. Visual inspection shows that there is a good concordance between estimated and measured values. As the lead time increases, a slightly increase in errors is noted. An evaluation of the model in statistical terms is presented in Table 1.

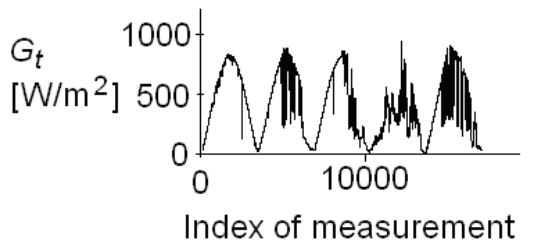

Fig.1 Time series of global solar irradiance. Data measured from $16^{\text {th }}$ to $20^{\text {th }}$ July 2010 and restricted to $h>5^{\circ}$ are considered.

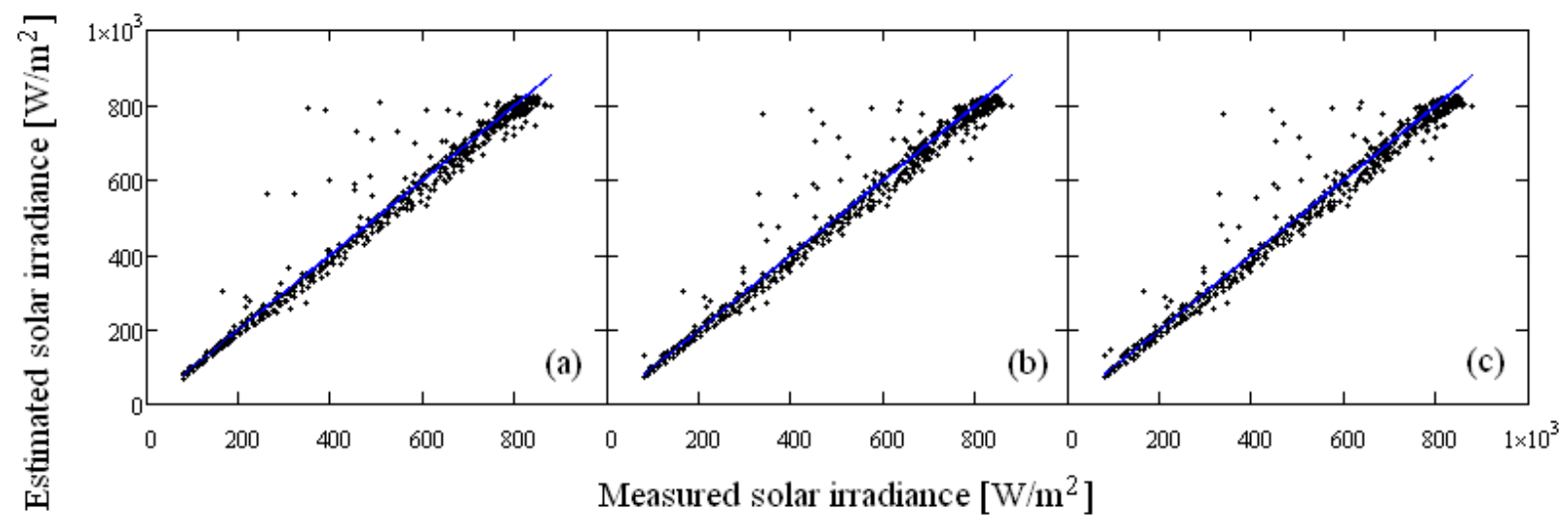

Fig.2. Estimated vs. measured solar irradiance at three different lead times: (a) 15 seconds; (b) 5 minutes; (c) 10 minutes

TABLE 1. Statistical indicators of accuracy for the estimation of solar irradiance with Eq. (4) and Eq. (5), respectively, at different lead time.

\begin{tabular}{|c|c|c|c|c|c|c|c|}
\hline \multirow{2}{*}{} & Lead time & \multicolumn{2}{|c|}{15 seconds } & \multicolumn{2}{c|}{5 minutes } & \multicolumn{2}{c|}{10 minutes } \\
\cline { 2 - 8 } & & $r M B E$ & $r R M S E$ & $r M B E$ & $r R M S E$ & $r M B E$ & $r R M S E$ \\
\hline $16-07-2010$ & Eq. (4) & 0.073 & 0.105 & 0.070 & 0.092 & 0.070 & 0.092 \\
\hline & Eq. (5) & -0.030 & 0.067 & -0.034 & 0.055 & -0.035 & 0.057 \\
\hline $17-07-2010$ & Eq. (4) & 0.042 & 0.064 & 0.055 & 0.118 & 0.055 & 0.118 \\
\hline & Eq. (5) & -0.015 & 0.050 & -0.003 & 0.103 & -0.003 & 0.103 \\
\hline $18-07-2010$ & Eq. (4) & 0.050 & 0.095 & 0.051 & 0.102 & 0.051 & 0.102 \\
\hline \multirow{2}{*}{$19-07-2010$} & Eq. (5) & 0.023 & 0.078 & 0.025 & 0.087 & 0.025 & 0.087 \\
\hline & Eq. (4) & 0.213 & 0.269 & 0.211 & 0.280 & 0.211 & 0.280 \\
\hline $20-07-2010$ & Eq. (5) & 0.026 & 0.124 & 0.016 & 0.148 & 0.017 & 0.149 \\
\hline & Eq. (4) & 0.045 & 0.152 & 0.035 & 0.116 & 0.035 & 0.116 \\
\hline & Eq. (5) & -0.002 & 0.142 & -0.011 & 0.109 & -0.011 & 0.109 \\
\hline
\end{tabular}


Several conclusions can be drawn from Table 1. At large lead times the model accuracy is saturated. In general, by applying the correction $\tau_{a}$ the model accuracy is substantially enhanced. This behavior is noted on both clear and variable sky days. For example, at lead time 5 minutes $r R M S E$ decrease from $9.2 \%$ to $5.5 \%$ in the clear sky day $16^{\text {th }}$ July and from $28.0 \%$ to $14.8 \%$ in the mostly cloudy day $19^{\text {th }}$ July. As the stability of the radiative regime decrease, the model accuracy decrease.

\section{Conclusions}

This paper is focused on improving the accuracy of the empirical clear sky solar irradiance models. A dynamic correction for the average atmospheric transmittance encapsulated in the model equation is tested. Overall results demonstrates that the proposed correction improve substantially the quality of solar irradiance estimates whatever is the radiative regime.

\section{References}

[1] Solar Radiation Monitoring Station of the West University of Timisoara, Romania http://solar.physics.uvt.ro/srms

[2] M. Kottek, J. Grieser, C. Beck, B. Rudolf, F. Rubel, Meteorologische Zeitschrift 15 (2006) 259.

[3] V. Badescu, Theoretical and Applied Climatology 72 (2002) 127.

[4] M Paulescu, V Badescu, Theoretical and Applied Climatology 103 (2011) 459.

[5] M. Paulescu, Z. Schlett, Renewable Energy 29 (2004) 767.

[6] M. Paulescu, Z. Schlett, Theoretical and Applied Climatology 75 (2003) 203.

[7] MathCAD (2014) PTC - MathCAD - Engineering Calculations Software. $<$ http://www.ptc.com/products/mathcad/>. 